Sampaio, NDS, Santos, MFA \& Paz, FAN (2020). Complications caused by nicotine during the pregnancy period. Research, Society and Development, 9(7): 1-18, e648974506.

\title{
Complicações causadas pela nicotina durante o período gestacional
}

Complications caused by nicotine during the pregnancy period

Complicaciones causadas por la nicotina durante el período de embarazo

Recebido: 13/05/2020 | Revisado:15/05/2020 | Aceito: 22/05/2020 | Publicado: 30/05/2020

Nadya Driely de Sousa Sampaio

ORCID: https://orcid.org/0000-0003-0071-3767

Centro Universitário Santo Agostinho, Brasil

E-mail: nadiadrielle@ hotmail.com

Mara França Alves dos Santos

ORCID: https://orcid.org/0000-0002-8732-6278

Centro Universitário Santo Agostinho, Brasil

E-mail:mhara.fhran30@gmail.com

Francisco Adalberto do Nascimento Paz

ORCID: https://orcid.org/0000-0001-6697-1705

Centro Universitário Santo Agostinho, Brasil

Email:pazadalberto19@hotmail.com

\section{Resumo}

O estudo objetivou analisar as produções científicas a cerca das complicações que o tabagismo exerce sobre a gravidez, investigar os problemas desenvolvidos na gestação devido ao uso do tabaco e verificar quais as medidas preventivas estão sendo tomadas para diminuir o uso do tabaco no período gravídico. Estudo de revisão integrativa da literatura, utilizando a estratégia PICo, atribuiu-se ao P: gestantes, ao I: complicações e ao Co: nicotina nas bases eletrônicas. Foram encontrados 11 artigos publicados entre 2015-2020. A primeira categoria abordou a incidência de gestantes fumantes. A segunda categoria identificou as Complicações de mãe gestantes fumantes. A terceira as Complicações apresentadas pelo RN. O presente estudo permite uma maior compreensão aos profissionais da saúde à cerca dos efeitos colaterais que o tabagismo tem sobre a gravidez e os problemas de desenvolvimento apresentado por fetos que tiveram contato com o fumo e o impacto que as politicas de prevenção ao tabagismo têm sobre as gestantes. 
Palavras-chave: Nicotina; Complicações; Gestação.

\begin{abstract}
The study aimed to analyze scientific productions about the complications that smoking has on pregnancy, investigate the problems developed during pregnancy due to tobacco use and verify what preventive measures are being taken to decrease tobacco use during pregnancy. An integrative literature review study, using the PICo strategy, was attributed to P: pregnant women, I: complications and Co: nicotine in electronic databases. There were 11 articles published between 2015-2020. The first category addressed the incidence of pregnant women who smoke. The second category identified the complications of pregnant smoking mothers. The third is the Complications presented by the RN. The present study allows health professionals to understand more about the side effects that smoking has on pregnancy and the developmental problems presented by fetuses who have had contact with smoking and the impact that smoking prevention policies have on women pregnant women.
\end{abstract}

Keywords: Nicotine; Complications; Gestation.

\title{
Resumen
}

El estudio tuvo como objetivo analizar las producciones científicas sobre las complicaciones que tiene fumar durante el embarazo, investigar los problemas desarrollados durante el embarazo debido al consumo de tabaco y verificar qué medidas preventivas se están tomando para disminuir el consumo de tabaco durante el embarazo. Un estudio integrador de revisión de literatura, utilizando la estrategia PICo, se atribuyó a P: mujeres embarazadas, I: complicaciones y Co: nicotina en bases de datos electrónicas. Hubo 11 artículos publicados entre 2015-2020. La primera categoría abordó la incidencia de mujeres embarazadas que fuman. La segunda categoría identificó las complicaciones de las madres embarazadas que fuman. El tercero son las complicaciones presentadas por el RN. El presente estudio permite a los profesionales de la salud comprender más acerca de los efectos secundarios que tiene el tabaquismo en el embarazo y los problemas de desarrollo que presentan los fetos que tuvieron contacto con el tabaquismo y el impacto que las políticas de prevención del tabaquismo tienen en las mujeres. mujeres embarazadas.

Palabras clave: Nicotina; Complicaciones; Gestación. 


\section{Introdução}

Resende (2017) afirma que a gestação é um momento único na vida da mulher, marcado por transformações fisiológicas que preparam o organismo para gerar o novo ser. É necessária a adoção de hábitos saudáveis, a fim de conservar a saúde materna e fetal, prevenindo e minimizando complicações durante e após a gestação.

Na gestação o tabagismo pode afetar o sistema imunológico da mãe, diminuindo o óxido nítrico. A ruptura prematura das membranas é secundária a toxicidade do fumo, o tabaco reduz a inativação do fator de ativação de plaquetas podendo provocar parto prematuro e diversas transformações placentário (Nunes et al. 2016).

Os efeitos do cigarro são cientificamente maléficos durante o período gestacional devido sua composição química. Porem nesse período é importante que a gestante interrompa o consumo desse tipo de substância. Algumas substâncias encontradas no cigarro como a nicotina têm possíveis ações maléficas para o bebê durante o período gestacional. A nicotina sabe-se que sua ação eleva o risco de recém-nascidos com baixo peso, natimortos e abortos espontâneos e sua absorção é realizada no trato respiratório. Autores relatam que o tabagismo causa diminuição da pressão de oxigênio materno, comprometendo assim a perfusão correta da placenta e a diminuição de absorção de nutrientes para o bebê (Lima et al. 2018).

Hábitos maternos prejudiciais ou nocivos à saúde, como o tabagismo, constituem um importante problema de saúde pública devido à sua elevada prevalência e impacto negativo nas condições de saúde do feto e do recém-nascido (RN), sobretudo relacionadas ao seu crescimento e desenvolvimento, (Teixeira, Lucena \& Echer, 2014).

Para com Borges (2018), além das alterações que o feto sofre dentro da barriga da mãe, após o nascimento, os bebês de mães fumantes podem apresentar, alterações dos sinais antropométricos como, por exemplo, altura e perímetro da cabeça. Além disso, casos mais graves como incidência de anomalias congênitas como fenda palatina e microcefalia.

Portanto, o controle da gestante usuária de cigarro deve ser iniciado por meio de estratégias voltadas para a detecção precoce nos serviços de saúde. Um dos problemas que o profissional de saúde se depara é, justamente, identificação das gestantes que fazem o uso do tabaco, uma vez que existe uma pressão social para cessar com o hábito de fumar, o que acaba por intimidar a mulher para revelar sua condição de pessoa tabagista. Sabe-se que elas tendem a não relatar o consumo dessas substâncias (Rocha et al. 2019).

A equipe multidisciplinar por meio do exame pré-natal, deve realizar a investigação do hábito de fumar da gestante e das pessoas que convivem com ela em seu ambiente familiar e 
profissional e, essa abordagem se constitui na base para ações a serem implementadas nas consultas, onde será orientada sobre os riscos do tabagismo, oferecendo ações de promoção da saúde física e mental e na redução de agravantes durante o período gestacional para a mãe e para o bebê, (Yabuuti \& Bernardy 2014).

O presente estudo objetivou analisar as produções científicas a cerca das complicações que o tabagismo exerce sobre a gravidez, investigar os problemas desenvolvidos na gestação devido ao uso do tabaco e verificar quais as medidas preventivas estão sendo tomadas para diminuir o uso do tabaco no período gravídico.

\section{Metodologia}

Foi realizado um estudo de revisão de literatura pelo método de revisão integrativa conduzida por seis etapas de investigação: identificação do tema e seleção da hipótese ou questão de pesquisa; estabelecimento de critérios de inclusão e exclusão de estudos/amostragem ou busca na literatura; definição das informações a serem extraídas dos artigos selecionados/categorização dos estudos; avaliação dos estudos incluídos na revisão integrativa; interpretação dos resultados e apresentação do revisão/síntese do conhecimento (Galvão, 2008).

Com referência a Carvalho (2010) trata-se de uma abordagem ampla do fenômeno investigado, visto que inclui estudos de diferentes abordagens metodológicas, além de incorporar uma gama de propósitos, como: definição de conceitos, revisão de teorias e evidências e análise de problemas metodológicos de um tema em particular.

Tem por finalidade a reunião e sintetização dos resultados de pesquisa sobre um determinado tema ou questão, conforme estratégia sistemática e ordenada, possibilitando a síntese do conhecimento, além de identificar lacunas que necessitam ser preenchidas por meio da realização de novos estudos (Polit \& Beck, 2006).

Para elaboração da questão de pesquisa será utilizada a estratégia PICo (P- Paciente, problema ou população; I- fenômeno de interesse; Co- Contexto). Comumente utilizada para realização de estudos não clínicos. Esse acrônimo pode ser empregado para construção de questões de naturezas variadas, possibilitando a definição correta de que informações são necessárias para a resolução da questão de pesquisa, além de maximizar a recuperação de evidências nas diversas bases de dados, foca o escopo da pesquisa e evita a realização de buscas desnecessárias (Lockwood et al. 2017). 
Atribuiu-se ao P: gestantes, ao I: complicações e ao Co: tabagismo. Foram utilizados como critérios de inclusão: estudos primários disponíveis na integra nas bases de dados e que retratassem resultados de estudos desenvolvidos com seres humanos. Aqueles cuja metodologia adotada permitisse obter evidências fortes: ensaios clínicos randomizados controlados individuais, estudos do tipo coorte, estudos com delineamento de pesquisa quase experimental e estudos transversais e longitudinais.

Já para os critérios de exclusão foi direcionado ao material publicado em anos anteriores à 2015, pois se entende a importância de apresentar discussões mais recentes, artigos que não abordavam diretamente a temática proposta, e textos que se encontravam incompletos; indisponíveis na íntegra on-line, que não forneciam informações suficientes acerca da temática do estudo.

A busca foi realizada por dois revisores, simultaneamente e de forma independente, no período de março a abril de 2020. Utilizaram-se as bases de dados: Literatura LatinoAmericana e do Caribe em Ciências da Saúde (LILACS) Medical Literature Analysis and Retrieval Sistem Online (MEDLINE/Pubmed). As estratégias de busca serão adaptadas para cada uma das bases de dados selecionadas, considerando suas especificidades de acesso. Os descritores selecionados para operacionalização das buscas encontrar-se-ão inseridos nos vocabulários Descritores em Ciências da Saúde (DeCS).

Para coleta de dados foi utilizado um instrumento elaborado pelos autores deste estudo, contendo informações acerca do ano de publicação, país de origem, área de atuação dos autores, detalhamento do método (tipo de estudo, amostra, cenário), intervenção e avaliação da intervenção.

\section{Resultados e Discussão}

A partir da leitura e análise do material encontrado, agruparam-se as informações relevantes dos artigos e organizaram-se em tabelas e quadros para uma melhor compreensão. Sendo a Tabela 1 a apresentação e distribuição das produções científicas de acordo com o período de publicação, a abordagem metodológica e, o periódico de publicação. 
Tabela 1 - Distribuição das produções científicas segundo o ano de publicação, abordagem metodológica e, periódico de publicação, $(\mathrm{n}=11)$. Teresina, 2020.

\begin{tabular}{|c|c|c|}
\hline VARIÁVEIS & $\mathbf{N}^{\mathbf{0}}$ & $\%$ \\
\hline \multicolumn{3}{|l|}{ ANO DE PUBLICAÇÃO } \\
\hline 2013 & 01 & 9,09 \\
\hline 2015 & 01 & 9,09 \\
\hline 2016 & 04 & 36,36 \\
\hline 2017 & 02 & 18,18 \\
\hline 2018 & 03 & 27,27 \\
\hline \multicolumn{3}{|l|}{ ABORDAGEM METODOLÓGICA } \\
\hline Quantitativa & 05 & 45,45 \\
\hline Revisão Integrativa & 03 & 27,27 \\
\hline Qualitativa & 03 & 27,27 \\
\hline \multicolumn{3}{|l|}{ PERIÓDICO DE PUBLICAÇÃO } \\
\hline Rev.O mundo da saúde & 01 & 9,09 \\
\hline Arq. Catarin Med & 01 & 9,09 \\
\hline Rev. REUOL & 01 & 9,09 \\
\hline Rev. Portuguesa de Enfermagem de Saúde Mental & 01 & 9,09 \\
\hline J Bras Pneumol & 01 & 9,09 \\
\hline Enfermagem Brasil & 01 & 9,09 \\
\hline Acta Paul Enferm & 01 & 9,09 \\
\hline Rev Enferm Atenção Saúde & 01 & 9,09 \\
\hline INFAD Revista de Psicología & 01 & 9,09 \\
\hline R. Enferm. UFJF & 01 & 9,09 \\
\hline Coodernação de pesquisa & 01 & 9,09 \\
\hline \multicolumn{3}{|l|}{ Fonte: Base de dados, 2020.} \\
\hline 6 & & \\
\hline
\end{tabular}


Research, Society and Development, v. 9, n. 7, e648974506, 2020

(CC BY 4.0) | ISSN 2525-3409 | DOI: http://dx.doi.org/10.33448/rsd-v9i7.4506

Quanto às modalidades de estudo encontradas nos artigos, observou-se que a que predominou foi a pesquisa de campo, seguida das de revisão integrativa.

Com a pesquisa, foram obtidos onze trabalhos científicos, que após terem sido lidos na íntegra, foram distribuídos em um quadro de forma resumida nas seguintes variáveis: autores, ano, objetivo, intervenções e os resultados (Quadro 1). Com ordem do número 01 ao 11 para facilitar a análise de identificação das etapas e agrupados em eixos temáticos.

Quadro 1 - (Caracterização das publicações científicas segundo autor, ano, objetivo, intervenções e os resultados).

\begin{tabular}{|c|c|c|c|}
\hline AUTOR, ANO & OBJETIVO & INTERVENÇÃO & RESULTADOS \\
\hline $\begin{array}{l}\text { Nunes e Campos } \\
\text { (2016) }\end{array}$ & $\begin{array}{l}\text { Avaliar o hábito tabácico a } \\
\text { fatores associados ao } \\
\text { tabagismo na gestação. }\end{array}$ & $\begin{array}{l}\text { A amostra foi de um } \\
\text { estudo transversal que } \\
\text { incluiu } 241 \text { puérperas no } \\
\text { serviço de ginecologia e } \\
\text { obstetrícia na } \\
\text { maternidade do hospital } \\
\text { de são José-Santa } \\
\text { Catarina- Brasil }\end{array}$ & $\begin{array}{l}\text { Foram analisados dados de } \\
\text { questionários de } 241 \\
\text { puérperas e de seus } \\
\text { respectivos RN, de agosto de } \\
\text { 2013. Dessas, } 152(63,1 \%) \\
\text { nunca fumaram, } 30(12,4 \%) \\
\text { fumavam e cessaram antes } \\
\text { de gestação; } \\
\text { (2,1\%)fumavam } \\
\text { esporadicamente, durante a } \\
\text { gestação , 16(6,6\%) } \\
\text { fumavam, mas reduziram o } \\
\text { cigarro ao saber que estavam } \\
\text { grávidas e 20(8,3\%) } \\
\text { participantes continuaram } \\
\text { fumando mesmo sabendo } \\
\text { que estavam grávidas. } \\
. \text { Dentre participantes,102(42,3\%) } \\
\text { pacientes referiram conviver } \\
\text { com fumantes em casa. }\end{array}$ \\
\hline $\begin{array}{l}\text { Lopes, } \\
\text { Tsunechiro, Pina- } \\
\text { Oliveira e Chiesa } \\
\text { (2015) }\end{array}$ & $\begin{array}{l}\text { Verificar o comportamento } \\
\text { dos fumantes na gestação } \\
\text { no que se refere: a } \\
\text { previdência do tabagismo; à } \\
\text { caracterização da história } \\
\text { tabagística da gestante, aos } \\
\text { níveis de dependência à } \\
\text { iniciativa e à relação entre o } \\
\text { número de apenas }\end{array}$ & $\begin{array}{l}\begin{array}{l}\text { Nessa amostra } \\
\text { puérperas }\end{array} \\
\text { entrevistadas, } \\
\text { novembro de } 2012 \text { a } \\
\text { janeiro de } 2013 \text { em uma } \\
\text { maternidade filantrópica } \\
\text { de São Paulo. }\end{array}$ & $\begin{array}{l}\text { Foi verificada a prevalência } \\
\text { de } 11 \% \text { de gestantes } \\
\text { fumantes, a maioria com } \\
\text { grau leve de dependência à } \\
\text { nicotina. A média encontrada } \\
\text { do consumo foi } 15,6 \text { anos. } \\
\text { Os recém- nascidos de } \\
\text { mulheres } \\
\text { apresentaram fumantes }\end{array}$ \\
\hline
\end{tabular}


Research, Society and Development, v. 9, n. 7, e648974506, 2020

(CC BY 4.0) | ISSN 2525-3409 | DOI: http://dx.doi.org/10.33448/rsd-v9i7.4506

\begin{tabular}{|c|c|c|c|}
\hline & $\begin{array}{l}\text { consumidor e o peso do } \\
\text { recém-nascido. }\end{array}$ & & $\begin{array}{l}\text { nascimento estatisticamente } \\
\text { menor do que o de mulher } \\
\text { não fumante. A abordagem } \\
\text { do pré-natal é insuficiente, } \\
35,9 \text { das mulheres não } \\
\text { souberam informar nenhuma } \\
\text { patologia associada ao } \\
\text { cigarro e ao bebê. }\end{array}$ \\
\hline $\begin{array}{l}\text { Santos Santana et } \\
\text { al. ( 2015) }\end{array}$ & $\begin{array}{l}\text { Analisar o conhecimento } \\
\text { das gestantes sobre o } \\
\text { maléfico uso do tabaco e } \\
\text { suas implicações para o } \\
\text { feto. }\end{array}$ & $\begin{array}{l}\text { Trata-se de um estudo } \\
\text { exploratório e descritivo } \\
\text { com abordagem } \\
\text { qualitativa em que os } \\
\text { dados foram coletados } \\
\text { em domicílios com } 13 \\
\text { gestantes fumantes } \\
\text { cadastradas USF. }\end{array}$ & $\begin{array}{l}\text { Os resultados revelaram que } \\
\text { as entrevistadas revelaram } \\
\text { um conhecimento parcial das } \\
\text { complicações do cigarro na } \\
\text { gravidez e, sendo a considera } \\
\text { o problema respiratório } \\
\text { como algo alarmante, daí ser } \\
\text { necessário as orientações } \\
\text { sobre o uso deste no } \\
\text { ciclogravítico-puerperal, } \\
\text { intensivo e de modo } \\
\text { persistente no pré-natal; }\end{array}$ \\
\hline AUTOR, ANO & OBJETIVO & INTERVENÇÃO & RESULTADOS \\
\hline $\begin{array}{l}\text { Bertani, Garcia, } \\
\text { Tanni e Godoy } \\
(2015)\end{array}$ & $\begin{array}{l}\text { Examinar o padrão de } \\
\text { consumo do tabaco e } \\
\text { conhecimentos sobre as } \\
\text { doenças relacionadas ao } \\
\text { tabaco. }\end{array}$ & $\begin{array}{lrr}\text { Consistiu em estudo } \\
\text { transversal com } & 61 \\
\text { gestantes em um hospital } \\
\text { universitário } & \text { em } \\
\text { unidades básicas } & \text { de } \\
\text { saúde em } & \text { Botucatu-SP. } \\
\text { Dados coletados } & \text { em } \\
\text { maio 2014, } & \text { de } \\
\text { entrevistas } & \\
\text { semiestruturada. }\end{array}$ & $\begin{array}{l}\text { Os resultados mostram que } \\
\text { das } 61 \text { gestantes avaliadas, } \\
25 \text { eram fumantes, média de } \\
\text { idade } 26,4 \text { ou mais ou menos } \\
7,4 \text { anos. E } 24 \text { eram ex- } \\
\text { fumantes e } 12 \text { nunca } \\
\text { fumaram. Das } 25 \text { fumantes, } \\
12 \text { relataram que o marido } \\
\text { fumava, e } 4 \text { relataram que } \\
\text { pelo menos um dos } \\
\text { moradores do domicílio } \\
\text { fumavam; a maioria das } \\
\text { fumantes e ex-fumantes não } \\
\text { conheciam os malefícios do } \\
\text { tabagismo para a saúde delas } \\
\text { nem para o recém -nascido. }\end{array}$ \\
\hline $\begin{array}{l}\text { Lucchese, } \\
\text { Paranhos, Netto, } \\
\text { Vera e Silva } \\
\text { (2016) }\end{array}$ & $\begin{array}{l}\text { Calcular a prevalência e } \\
\text { analisar os fatores } \\
\text { associados ao uso nocivo de } \\
\text { tabaco uma vez na vida e }\end{array}$ & $\begin{array}{l}\text { Foi realizado um estudo } \\
\text { transversal em } 330 \\
\text { gestantes atendidas em } \\
\text { Centros especializados }\end{array}$ & $\begin{array}{l}\text { Ao término, foram } \\
\text { entrevistadas } 334(92,6 \%) \text { da } \\
\text { amostra gestantes, houve } \\
\text { perda de 27indivíduos, }\end{array}$ \\
\hline
\end{tabular}


Research, Society and Development, v. 9, n. 7, e648974506, 2020

(CC BY 4.0) | ISSN 2525-3409 | DOI: http://dx.doi.org/10.33448/rsd-v9i7.4506

\begin{tabular}{|c|c|c|c|}
\hline & $\begin{array}{l}\text { verificar o seu uso nocivo } \\
\text { entre gestantes. }\end{array}$ & $\begin{array}{l}\text { no atendimento à saúde } \\
\text { da mulher na atenção } \\
\text { básica. } 361 \text { indivíduos } \\
\text { representou a população } \\
\text { em foco. Os dados foram } \\
\text { coletados de maio de } \\
2014 \text { a outubro de } 2015 . \\
\text { Os dados dos } \\
\text { antecedentes pessoais } \\
\text { /familiares e o } \\
\text { rastreamento de uso de } \\
\text { tabaco foram obtidos por } \\
\text { meio de questionário } \\
\text { sóciodemográfico. }\end{array}$ & $\begin{array}{l}\text { houve acréscimo de 20\%, } \\
\text { mas não comprometeu o } \\
\text { cálculo amostral. A idade } \\
\text { média das gestantes de } 24,3 \text {, } \\
\text { e a prevalência de uso de } \\
\text { tabaco na gestação } 123 \text { ou } \\
34,1 \% \text { o 'uso nocivo' } 32 \text { ( } \\
9 . \% \text { ). Na análise múltipla o } \\
\text { desfecho uso nocivo foi } \\
\text { associado a antecedentes } \\
\text { familiares de tabagismo e } \\
\text { uso de álcool .das } 124 \\
\text { mulheres exposta ao uso de } \\
\text { tabaco, } 45 \text { desenvolveram o } \\
\text { habito de fumar, mas } 13 \\
\text { suspenderam por conta da } \\
\text { gestação e } 32 \text { mantiveram o } \\
\text { hábito. }\end{array}$ \\
\hline $\begin{array}{l}\text { dos Reis, Cunha e } \\
\text { Garcia (2016) }\end{array}$ & $\begin{array}{l}\text { Identificar as consequências } \\
\text { do uso do tabaco durante a } \\
\text { gestação e os efeitos para o } \\
\text { recém- nascido. }\end{array}$ & $\begin{array}{l}\text { Trata-se de uma revisão } \\
\text { de literatura de caráter } \\
\text { exploratório, adotou-se } \\
\text { como análise de dados a } \\
\text { técnica de fichamento e } \\
\text { planilhas. }\end{array}$ & $\begin{array}{l}\text { O cigarro causa grave } \\
\text { consequências ao organismo } \\
\text { humano, principalmente no } \\
\text { sistema nervoso e pulmonar. } \\
\text { O uso do tabaco em } \\
\text { gestantes acarreta sérias } \\
\text { implicações para a saúde, o } \\
\text { crescimento e o } \\
\text { desenvolvimento do recém- } \\
\text { nascido, podendo } \\
\text { permanecer até a fase adulta. }\end{array}$ \\
\hline $\begin{array}{l}\text { Machado, Borges } \\
\text { e de Resende } \\
\text { (2017) }\end{array}$ & $\begin{array}{l}\text { Apresentar as evidências } \\
\text { disponíveis na literatura } \\
\text { nacional sobre as principais } \\
\text { complicações apresentadas } \\
\text { por recém-nascidos (RNs) } \\
\text { de mães tabagistas no } \\
\text { período neonatal. }\end{array}$ & $\begin{array}{l}\text { Fez- se uma revisão de } \\
\text { artigos organizados por } \\
\text { meio de formulários para } \\
\text { coleta de dados. }\end{array}$ & $\begin{array}{l}\text { Os resultados apontam para a } \\
\text { coleta de artigos entre } 2006 \text { a } \\
2016 \text { com as seguintes } \\
\text { categorias: a primeira a } \\
\text { incidência do tabagismo na } \\
\text { gestação e a segunda: } \\
\text { complicações apresentadas } \\
\text { pelo RN. }\end{array}$ \\
\hline AUTOR, ANO & OBJETIVO & INTERVENÇÃO & RESULTADOS \\
\hline $\begin{array}{l}\text { Martins, Cruz, } \\
\text { Pereira e Lima } \\
(2018)\end{array}$ & $\begin{array}{l}\text { Descrever as complicações } \\
\text { na gravidez relacionadas ao } \\
\text { tabagismo }\end{array}$ & $\begin{array}{lr}\text { Fez-se se } & \text { um } \\
\text { levantamento } & \\
\text { bibliográfica com dados } \\
\text { de artigos sobre } \\
\text { complicações } & \text { de }\end{array}$ & $\begin{array}{l}\text { Os estudos apresentam haver } \\
\text { o risco do uso do tabaco } \\
\text { durante a gravidez associam- } \\
\text { se as variáveis: condições }\end{array}$ \\
\hline
\end{tabular}


Research, Society and Development, v. 9, n. 7, e648974506, 2020

(CC BY 4.0) | ISSN 2525-3409 | DOI: http://dx.doi.org/10.33448/rsd-v9i7.4506

\begin{tabular}{|c|c|c|c|}
\hline & & $\begin{array}{l}\text { mulheres gestantes } \\
\text { fumantes do período de } \\
2012 \text { a } 2018 \text { no Brasil. }\end{array}$ & financeiras e escolaridade. \\
\hline $\begin{array}{l}\text { Coutinho et al. } \\
2018\end{array}$ & $\begin{array}{l}\text { Identificar os } \\
\text { determinantes do consumo } \\
\text { de tabaco e vigilância na } \\
\text { gravidez. }\end{array}$ & $\begin{array}{l}\text { As participantes } \\
3232 \text { mulheres, estudo } \\
\text { realizado } 32 \\
\text { maternidades, de Norte e } \\
\text { do Sul de Portugal .o } \\
\text { instrumento de colheita } \\
\text { de dados foi o } \\
\text { questionário que } \\
\text { permitiu a caracterização } \\
\text { sóciodemográfica com a } \\
\text { idade das mulheres - } \\
28,75 \\
\text { anos,escolaridade78.2\% } \\
\text { de nacionalidade } \\
\text { portuguesa e } 21,8 \% \\
\text { estrangeiras. }\end{array}$ & $\begin{array}{l}\text { Em relação ao consumo de } \\
\text { tabaco durante a gravidez, } \\
\text { verifica-se nas mulheres } \\
\text { fumadoras, as percentagens } \\
\text { elevadas corresponde às que } \\
\text { não possuem formação } \\
\text { profissional } 63,8 \% \text {, } \\
\text { secundadas pelas } 24,7 \% \text { das } \\
\text { que possuem formação } \\
\text { superior .E que há uma } \\
\text { probabilidade de } 72,3 \% \text { de } \\
\text { mulheres a efetuarem } \\
\text { vigilância adequada da } \\
\text { gravidez e de não terem } \\
\text { fumado durante a mesma, } \\
\text { enquanto } 27,7 \% \text { não tiveram } \\
\text { vigilância pré-natal adequada } \\
\text { e fumaram durante a } \\
\text { gravidez. }\end{array}$ \\
\hline $\begin{array}{l}\text { Carvalho et al. } \\
2018\end{array}$ & $\begin{array}{l}\text { Identificar as dificuldades } \\
\text { para a cessação do hábito } \\
\text { de fumar durante a } \\
\text { gestação. }\end{array}$ & $\begin{array}{l}\text { As participantes da } \\
\text { pesquisa foram quatro } \\
\text { gestantes que faziam uso } \\
\text { tabaco ,usuária da UBS, } \\
\text { maior de } 18 \text { anos e que } \\
\text { estavam realizando o } \\
\text { pré-natal - }\end{array}$ & $\begin{array}{l}\text { A idade das mulheres } \\
\text { entrevistadas variou de } 18 \text { a } \\
26 \text { anos; tempo de gestação } \\
\text { entre } 5 \text { a } 8 \text { meses. Revelaram } \\
\text { que eram tabagistas há mais } \\
\text { de três anos observaram-se } \\
\text { duas categorias de análise; } \\
\text { motivo para a gestante fumar } \\
\text { versus motivo para deixar de } \\
\text { fumar: a ótica da gestante; } \\
\text { cessação do hábito de fumar: } \\
\text { tentativas sem resultado. }\end{array}$ \\
\hline
\end{tabular}

Fonte: Base de dados, 2020.

Foram selecionados os 11 artigos, realizou-se a definição das principais características das produções científicas do consumo de tabaco em mulheres grávidas categorizados em 3 (três) eixos que foram utilizados para nortear a realização da pesquisa, conforme Quadro 2. 
Research, Society and Development, v. 9, n. 7, e648974506, 2020

(CC BY 4.0) | ISSN 2525-3409 | DOI: http://dx.doi.org/10.33448/rsd-v9i7.4506

Quadro 2 - Distribuição dos artigos de acordo com as categorias.

\begin{tabular}{|l|l|l|}
\hline Categorias & Frequência & Porcentagem \\
\hline A incidência de gestantes fumantes & 06 & $54,54 \%$ \\
\hline Complicações de mãe gestantes fumantes & 03 & $27,27 \%$ \\
\hline Complicações apresentadas pelo RN & 02 & $18,18 \%$ \\
\hline
\end{tabular}

Fonte: Base de dados, 2020.

Considerando a categoria referente à incidência de gestantes fumantes foi constatada em 06 (seis) artigos. Correspondendo cerca de 54,54\% dos artigos pesquisados.

Nos 02 (dois) estudos, Bertani et al. (2015) e Lucchese et. al (2016) relatam sobre a prevenção do tabagismo na gravidez .Observou-se que foi elaborado através de uma coleta com 61 gestantes em Botucatu-SP, apontou-se que 25 (40,9\%) eram fumantes .Coletou-se nessas entrevistadas a que são fumantes passivas, cerca de 39 (63,9\%) das gestantes e foram observados a cerca do conhecimento sobre o malefício do cigarro ,das 49 pesquisadas apenas $23(39,3 \%)$ acreditam que fumar não prejudica o recém -nascido e 21 (42,9\%) consumiram álcool durante a gestação e 18 (36,7\%) disseram aumentar o consumo de cigarro ao beber, (Ibidem, 2015).

O estudo foi realizado com 330 gestantes, com idade média de 24,3, e nessa coleta identificou-se sobre o uso de tabaco que 124( $37,1 \%$ ) fumou um vez na vida. Dessa probabilidade o histórico delas advém de familiares com uso de álcool e fumo, e desse resultado 45 mostraram o desenvolvimento do hábito de fumar, sendo que 13 suspenderam por haver engravidado. Já as consideradas com "uso nocivo", 32 (9,6\%), mantiveram o hábito mesmo gestante, sendo que 23 gestantes apresentaram dependência médio do tabaco e 9 com nível elevado (Lucchese, et al. 2016).

$\mathrm{Na}$ pesquisa coletaram-se instrumentos sobre a caracterização do consumo de tabaco em mulheres grávidas. Verificou-se em literatura que há uma tendência, no uso do tabaco na gravidez, de haver uma associação às varáveis sociodemográficas como nível de escolaridade baixa, ou elevados níveis de escolaridade, baixo nível de economia bem como pela coabitação com fumadores, e ainda estudos apresentam haver uma associação entre os sintomas 
depressivos pré-natais e/ou durante a gravidez e a dependência da nicotina (André et al. 2015).

\section{1 - Incidência de gestantes fumantes}

Um estudo realizado em uma maternidade filantrópica de Município de São Paulo entrevistaram 273 puérperas. Tal investigação revelou a prevalência de $11 \%$ de gestantes fumantes, sendo constatado que a maioria revelou um grau leve de dependência à nicotina. Muitas iniciaram o consumo de fumo numa média de idade de 15,6 anos. No estudo, os recém-nascidos apresentaram o peso menor em relação aos filhos de não fumantes. E ainda revelou-se nessa pesquisa que o conhecimento da gestante em relação ao tabagismo e à saúde do bebê é baixo (Lopes et al. 2015).

A pesquisa ocorreu por meio de entrevista realizada com quatro mulheres gestantes e que utilizavam tabaco, entre 18 a 26 anos, com tempo de gestação entre 5 a 8 meses, tabagista há mais de três anos, tais gestantes revelam não conseguir para de fumar e há tentativas sem resultados, até se mencionou como motivo o fato de morarem ou conviverem com pessoas fumantes. Observou-se que estudos apontam haver a incidência do tabagismo entre mulheres em idade fértil. É de grande importância, pois em consenso todos associam que o hábito de fumar na gravidez é um ato nocivo tanto para a gravidez quanto para o feto, sendo responsável por $20 \%$ dos fetos com baixo peso ao nascer, mesmo assim muitas mães não estão atentas a tais dados (Carvalho et al. 2018).

Por meio de uma amostragem realizada com 3232 mulheres que foram mãe com idade média de 28,75 anos, obtiveram-se dados interessantes em relação ao tema. Tais estudos, feitos com mulheres que moram em Portugal há mais de 7 meses, privilegiam a vigilância na gravidez, voltada para a gravidez sem tabaco com a precaução para evitar complicações na gravidez. Os dados revelam $75,2 \%$ efetiva vigilância foi feita na gravidez e não fizeram uso do cigarro, no entanto $27,7 \%$ não tiveram uma vigilância pré-natal adequada e fumaram durante a gravidez. Fatores como viver com o marido, sem planejar a gravidez, a probabilidade aumenta em vigiar o consumo de tabaco durante a gravidez, já as que moram na zona rural, com formação profissional ou técnica, que não exercem profissão, e com problemas na gravidez, possuem mais possibilidade de não vigiar a gravidez consumindo o tabaco, há diminuição (Coutinho et al. (2018).

Encontrou-se na leitura feita na literatura que, ao longo dos últimos anos, ocorreram poucas reduções no percentual de gestantes fumantes, ao se considerar somente as que 
fumam, pouca delas conseguiu parar de fumar nas semanas antecedentes ao parto. $\mathrm{Na}$ literatura observou-se haver ao longo dos últimos anos uma pequena redução no percentual de gestantes fumantes e ao se levar em consideração apenas as gestantes que fumam, uma parte delas conseguiu cessar o fumo durante a gravidez e outras não tiveram sucesso. A literatura observou haver mais frequência de grávidas fumantes usuárias de álcool, observou-se que a incidência do uso do fumo serve de porta para outras drogas (Lucchese et al. 2016).

\section{2 - Complicações de mãe gestantes fumantes}

A segunda categoria possibilitou por meio do estudo, analisar três (3) artigos ou (27,27\%) dos artigos coletados. Os valores encontrados no estudo aproximam-se da literatura. Nesta categoria identificaram-se as principais complicações apresentadas pelas mães gestantes tabagistas (Martins et al. 2018).

Este estudo ao relacionar o uso de tabagismo à gravidez enfatiza as complicações pelo fato de haver várias transformações fisiológicas e psicológicas no ciclo gestacional e com isso o sistema imunológico da mãe sofre, sendo afetada pela diminuição do óxido nítrico placentário, redução do fator e ativação de plaquetas e com isso provoca-se o parto prematuro. E ainda são apontados sobre o uso da nicotina haver uma redução do crescimento intra uterino, bem como o comprometimento do crescimento pulmonar alterando as funções respiratórias. E por haver uma ligação do monóxido de carbono à hemoglobina materna e fetal com a produção da carboxihemoglobina, acaba-se por se elevar os níveis nessa circulação e provocando hipóxia tecidual, isso aumenta o risco de enfarto cerebral do neonato e insuficiência placentária. Tais riscos, segundo os autores, associam-se às condição de vida em que as mesmas vivem como também pela escolaridade. E como agravante o convívio com pessoas que fumam, ficam mais expostas ao tabaco. Este estudo revelou que no Brasil há cerca de $9,14 \%$ de grávidas fumantes no Brasil, há riscos tanto para a mãe como para o feto, (Martins et al. 2018).

Estudos mostram que o tabagismo causa diminuição da pressão de oxigênio materno, compromete a perfusão adequada da placenta e absorção de nutrientes do feto. Tal estudo revelou que há danos na mãe gestante e no recém -nascido trazendo riscos de infarto cerebral no neonato, insuficiência placentária ,parto prematuro, pré-eclampsia, aborto prematuro gestação ectópica (Ibid et al. 2018). 
No estudo através de uma coleta de domicílios de 13 gestantes fumantes, revelou que essas mães têm pouco conhecimento das complicações do uso do cigarro na gravidez tanto para elas como para o feto. Ainda assim, ao serem entrevistadas as mães revelaram, acerca do conhecimento parcial que as mesmas têm sobre os malefícios do cigarro, referiram-se aos problemas respiratórios como alarmantes. Dentre as consequências observadas neste estudo foi apresentado o risco de abortos espontâneos, maior incidência de ruptura das membranas ovulares, placenta prévia, deslocamento prematuro da placenta, polidrâmio, sangramentos vaginais, falta de apetite devido à carência de vitamina B1 e ainda a elevação de pressão arterial e frequência cardíaca, retenção de líquidos e outros problemas. Na leitura 23,2\% são mães gestantes fumantes e fumam até o fim da gestação, (SANTOS, et al. 2015).

Em outro estudo com 241 grávidas puérperas jovens, com idade entre 18 e 24 anos, com risco no recém- nascido, prematuridade, baixo peso ao nascer e baixo perímetro cefálico, sendo que o tabagismo materno ativo foi $24,5 \%$ e passivo $42,3 \%$ e essas fumante fizeram menos consultas pré-natais, muitas com medo do julgamento por serem fumantes, ou por não querer cessar o tabagismo no momento e outras por falta de conhecimento por parte dos familiares de que o fumo passivo também causa complicações ao feto (Nunes \& Campos 2016).

\section{3 - Complicações apresentadas pelo $\mathbf{R N}$}

Nesta categoria foram cerca de $18,18 \%$ dos artigos(2). Nesta categoria elencaram-se as principais complicações apresentadas pelo RN de mães tabagistas.

Este estudo apresentou coletas de artigos que revelaram como principais complicações encontradas nos RNs foram: diminuição do peso, comprimento e perímetro cefálico e peso insuficiente ao nascer, comparando-se aos recém -nascidos de mães que nunca usaram tabaco (Machado, Borges \& Resende, 2017).

Nesta pesquisa há uma explanação sobre as sérias implicações para a saúde do RN , além de afetar o crescimento do recém- nascido, o desenvolvimento e pode perdurar até a vida adulta. Neste estudo as pesquisa a observam que o primeiro trimestre da gravidez, o cigarro possibilita que as substâncias advindas do tabaco chegam ao feto pela placenta, com isso justifica-se haver o deslocamento prematuro da placenta, placenta prévia, ruptura prematura da membrana, aborto espontâneo, prematuridade do recém-nascido de baixo peso e pequeno para a idade gestacional, observou-se ainda a síndrome da morte súbita em lactente, 
e ainda as complicações respiratórias e comportamentais na infância (Reis, Cunha \& Garcia, 2016).

As consequências no organismo do recém-nascido são diversas como: pneumonia, bronquite, e otite, ainda há riscos de leucemia na infância. E os autores enfatizam que as substâncias existentes no cigarro percorrem o organismo e do mesmo modo a nicotina que concentra no líquido amniótico, no leite materno, acaba lesando o recém-nascido no seu desenvolvimento. Há que se observar que os autores assinalam que na fase pós-natal estudouse o retardamento do desenvolvimento do sistema nervoso central e do trato gastrointestinal, sendo assim é aumentado o risco de cólica infantil à nicotina (Reis et al. 2016).

Os valores mencionados no estudo se mostraram próximos da literatura. Há casos que podem, não ser fidedignos, pelo fato de a mulher ser temente a reprovação dos pesquisadores, muitas desconhecem e tem pouca noção de que podem prejudicar os recém-nascidos com esse hábito de fumar. E apesar do fumo ter sido consumido em quantidade severa e em outras menos severas durante todo o período gestacional, não houve uma associação significativa entre o consumo de fumo durante a gestação e a presença de malformações fetais, (Machado, Borges \& Resende, 2017).

Os dados foram analisados de forma descritiva e os resultados apresentados e discutidos em categorias temáticas que levaram em consideração a similaridade de informações que responderão ao problema deste estudo.

\section{Considerações Finais}

Deixar o hábito de fumar é necessário em qualquer fase da vida, em especial na gestação. Anular o tabagismo é a forma mais eficaz de se evitar possíveis complicações para o feto e a gestante. Existe a necessidade de políticas públicas de combate ao tabagismo, voltadas especificamente para as gestantes, na tentativa de conscientizar a todos que o hábito de fumar pode contribuir para algumas malformações, principalmente na região da cabeça e do pescoço. Portanto, a redução e cessação do tabagismo na gravidez requerem esforços do próprio indivíduo, da sociedade, da família e dos profissionais envolvidos.

A gestação proporciona uma grande oportunidade para a promoção da cessação do tabagismo e deve ser vista como uma das melhores oportunidades pelo enfermeiro para fornecer mais informações sobre os malefícios do tabagismo para a saúde e oferecer tratamento para a sua cessação nas gestantes. Realizar programas educacionais que incluam 
informações sobre as consequências de todas as formas de uso do tabaco, utilizando novas formas que sejam eficazes para essa população específica.

Por meio de artigos estudados foi possível verificar que existe poucos trabalhos publicados na literatura nacional sobre o uso do tabaco no período gestacional e suas complicações, sendo necessária a realização de novas pesquisas a fim de facilitar a implementação de intervenções na assistência à saúde materna e do RN.

\section{Referências}

André MC, Cândido AE, Santiago C, Cru O, Carreira TM \& Amendoeira J. (2015). Consumo de tabaco na mulher grávida: Revisão sistemática da literatura. Revista Portuguesa de Enfermagem de Saúde Mental, (SPE2), 113-8.

Bertani AL, Garcia T, Tanni SE \& Godoy I. (2015). Prevenção do tabagismo na gravidez: importância do conhecimento materno sobre os malefícios para a saúde e opções de tratamento disponíveis. Jornal Brasileiro de Pneumologia, 175-81.

Coutinho E, Pereira C, Nelas P, Chaves C, Amaral O \& Cruz C. (2018). Determinantes do consumo de tabaco durante a gravidez e vigilância de gravidez. Revista INFAD de Psicología. International Journal of Developmental and Educational Psychology., 3(1), 317-24.

Da Silva M, Sousa Sombra IV, Silva JS, Silva JCB, Melo Dias LRF, Ferreira Calado RS ... \& de Menezes Silva GA. (2019). Aromaterapia para alívio da dor durante o trabalho de parto. Journal of Nursing UFPE/Revista de Enfermagem UFPE, 13(2).

dos Reis, M. A., da Cunha, P. R., \& Garcia, T. F. (2016). Consequências patológicas para os recém-nascidos advindos de gestantes tabagistas. Enfermagem Brasil, 15(5), 267-272.

Durieux, N., Vandenput, S., \& Pasleau, F. (2013). Médecine factuelle: La hiérarchisation des preuves par le Centre for Evidence-Based Medicine d'Oxford. Revue Médicale de Liège, 68(12), 644-649. 
Lopes, N. M. C., Tsunechiro, M. A., Pina-Oliveira, A. A., \& Chiesa, A. M. (2015). Tabagismo entre gestantes atendidas em maternidade filantrópica do Município de São Paulo. O Mundo da Saúde, São Paulo, 39(1).

Lockwood, C., Porrit, K., Munn, Z., Rittenmeyer, L., Salmond, S., Bjerrum, M., ... \& Stannard, D. (2017). Chapter 2: Systematic reviews of qualitative evidence. Joanna Briggs Institute reviewer's manual. The Joanna Briggs Institute.

Lucchese, R., Paranhos, D. L., Netto, N. S., Vera, I., \& Silva, G. C. (2016). Fatores associados ao uso nocivo do tabaco durante a gestação. Acta Paulista de Enfermagem, 29(3), 325-331.

Martins, A., Cruz, A., Pereira, S., \& Lima, E. (2018). Complicações na gravidez relacionadas ao tabagismo. Anais Concifa, 1(1).

Machado, M. B., Borges, J. P. A., \& de Resende, T. C. (2017). Complicações apresentadas por recém-nascidos de mães tabagistas no período neonatal. Revista de Enfermagem e Atenção à Saúde, 6(2).

Maria, F. N., Jornada, L. K., Sakae, T. M., Cassol Jr, O. J., Sakae, D. Y., \& Quevedo, J. L. (2016). Uso de álcool e tabaco por gestantes em maternidade do sul de Santa Catarina. Arquivos Catarinenses de Medicina, 44(1), 41-61.

Mendes, K. D. S., Silveira, R. C. D. C. P., \& Galvão, C. M. (2008). Revisão integrativa: método de pesquisa para a incorporação de evidências na saúde e na enfermagem. Texto \& Contexto-Enfermagem, 17(4), 758-764.

Nunes, R. D., \& de Campos, A. C. C. (2016). Avaliação do hábito tabágico e fatores associados ao tabagismo na gestação. Arquivos Catarinenses de Medicina, 44(3), 23-36.

Polit, D. F., \& Beck, C. T. (2006). Using research in evidence-based nursing practice. Essentials of nursing research. Methods, appraisal and utilization. Philadelphia (USA): Lippincott Williams \& Wilkins, 12, 457-94. 
Rocha, P. C., Britto e Alves, M. T. S. S. D., Chagas, D. C. D., Silva, A. A. M. D., Batista, R. F. L., \& Silva, R. A. D. (2016). Prevalência e fatores associados ao uso de drogas ilícitas em gestantes da coorte BRISA. Cadernos de Saúde Pública, 32, e00192714.

Rodrigues, A. L., de Souza, D. R., \& de Lima Borges, J. (2018). Consequências do uso de álcool e cigarro sobre o binômio mãe-feto . DêCiência em Foco, 2(1), 53-62.

Santos, H. T. S., Oliveira, G. S., Soares, P. C. F., Araújo, W. A., Almeida, E. U. A., \& Oliveira, M. M. L. R. (2015). Os malefícios do uso do tabaco na gestação e suas complicações ao feto. Rev Enferm UFPE Online, 9(9), 9978-82.

Souza, M. T. D., Silva, M. D. D., \& Carvalho, R. D. (2010). Revisão integrativa: o que é e como fazer. Einstein (São Paulo), 8(1), 102-6.

Teixeira, C. D. C., Lucena, A. D. F., \& Echer, I. C. (2014). Ações da equipe de saúde para gestantes e puérperas tabagistas. Revista Latino-Americana de Enfermagem, 22(4), 621-8.

Yabuuti, P. L. K., \& Bernardy, C. C. F. (2014). PERFIL de gestantes usuárias de drogas atendidas em um centro de atenção psicosocial. Revista Baiana de Saúde Pública, 38(2), 34456.

\section{Porcentagem de contribuição de cada autor no manuscrito}

Nadya Driely de Sousa Sampaio - 33,3\%

Mara França Alves dos Santos - 33,3\%

Francisco Adalberto do Nascimento Paz - 33,3\% 